\title{
Design of user-centered technologies to facilitate the mobilization of social media data
}

\author{
Adriana Alvarado Garcia
}

Published: 30 November 2021

\begin{abstract}
My research aims to address the challenges involved in using social media data within institutional contexts such as non-governmental organizations that focus on facing human rights crises through programmatic and legal interventions. The central question guiding my research is: How do we design human-centered technologies that facilitate the migration of social media data flows from online communities to legal and political contexts that support the defense of human rights and institutional change? To answer this question, I propose the design and development of a set of computational and qualitative tools that will allow organizations to make use of social media data as evidence to inform their work.
\end{abstract}

\section{Keywords:}

Social media data; Human-centered computing; Critical data studies; NGO; Civic technology.

\section{Introducción}

Las plataformas de redes sociales se han convertido en un mecanismo establecido para que activistas y ciudadanos recopilen información y se organicen frente al colapso político e institucional. Durante los últimos diez años, el contenido generado por los usuarios o datos de redes sociales han sido útiles tanto para identificar casos de violencia contra poblaciones específicas en ausencia de registros oficiales, como para ampliar las categorías de datos recopilados por los gobiernos durante situaciones de crisis humanitarias. Estos tipos de uso demuestran que el contenido generado por el usuario tiene el potencial de proporcionar información sobre las narrativas locales, las capacidades y la experiencia de los ciudadanos, lo cual puede ser útil para las Organizaciones No Gubernamentales (ONGs) que buscan reducir el impacto de las crisis de derechos humanos y comunitarias.

Sin embargo, a pesar del inmenso valor que posee la práctica de recurrir a los datos de las redes sociales para comprender los derechos humanos y las crisis comunitarias, existen tres desafíos

Alvarado Garcia, Adriana

Georgia Institute of Technology (Georgia Tech)

Atlanta, Georgia

adriana.ag@gatech.edu persistentes, los cuales impiden que este tipo de contenido se use como evidencia.

El primer desafío es la dificultad que implica determinar la veracidad de los datos de redes sociales. Dado que el contenido generado por el usuario no está estructurado y es difícil de verificar, es necesario desarrollar mecanismos para garantizar la calidad y confiabilidad de los datos [4]. El segundo desafío es retener el contexto del contenido generado por el usuario al reutilizarlo fuera de su lugar de producción. Una vez que este tipo de contenido se remueve de la plataforma donde se generó, las experiencias documentadas pierden significado y valor [4]. La tercera limitante se relaciona con la limitada representación de perspectivas y necesidades que se observan dentro de conjuntos de datos de redes sociales. Dependiendo de las herramientas y plataformas que utilizamos para recopilar datos, es posible observar en mayor o menor medida la representación de ciertos grupos de población [4]. Por último, el uso de contenido generado por el usuario para informar las prácticas de las ONG es una tarea desafiante porque la definición de evidencia varía según el contexto en el que se encuentran los hechos, los objetivos de la organización y la fuente de información. Por lo tanto, cualquier evidencia obtenida del contenido generado por el usuario se considera el punto de partida de las investigaciones en lugar de la conclusión [6].

Dentro de la comunidad de CSCW y HCI, ha habido un creciente interés por el uso de contenido generado por el usuario como una fuente de datos para analizar comportamientos, pensamientos y emociones de las personas para luego usarse como evidencia para caracterizar, cuantificar y predecir condiciones de salud, evaluar la frecuencia de actividades delictivas, y entender movimientos sociales. Sin embargo, la mayor parte de la investigación existente se centra en examinar el contenido de las comunidades en línea en lugar de analizar la interacción entre el comportamiento en línea y fuera de línea de esas comunidades. Por lo tanto, dentro de la disciplina de HCI aún queda pendiente por entender como diseñar herramientas que faciliten la movilización de los datos de las redes sociales desde su plataforma de origen a contextos institucionales, como las $\mathrm{ONG}$, para usarlos como evidencia y para informar sus intervenciones en las comunidades.

Mi investigación tiene como objetivo diseñar, implementar y evaluar herramientas que ayuden a solucionar los cuatro desafíos mencionados anteriormente para facilitar la migración de datos de las plataformas de redes sociales a contextos institucionales. Esta migración de datos es crucial para apoyar a las ONG que se enfocan en enfrentar crisis de derechos humanos a través de intervenciones programáticas y legales.

La pregunta central que guía mi investigación es: ¿Cómo diseñamos tecnologías centradas en el ser humano que faciliten 
la migración de los flujos de datos de las redes sociales desde las comunidades en línea a contextos legales y políticos que apoyan la defensa de los derechos humanos y el cambio institucional? Para responder a esta pregunta, propongo el diseño de herramientas contextualizadas que ayudaran a las ONG a utilizar datos de redes sociales para identificar capacidades locales, monitorear los derechos humanos y las crisis comunitarias, y desarrollar respuestas basadas en datos. Específicamente, el resultado de mi investigación será un conjunto de herramientas computacionales y cualitativas que ayudarán a las $\mathrm{ONG}$ en su proceso de (i) integrar la información de las comunidades detrás de los datos de las redes sociales y sus contrapartes en entornos fuera de línea para mantener la localidad de los datos, (ii) evaluar el enfoque más apropiado para evaluar la validez y precisión de los datos según el contexto y el tipo de datos, y (iii) resolver los desafíos de precisión, contexto y representación al aprovechar los datos de las redes sociales.

\section{Trabajo Relacionado}

Para las ONG de derechos humanos, el núcleo de su trabajo es utilizar los sistemas legales nacionales e internacionales para responsabilizar a los perpetradores por las violaciones de derechos humanos [5,6]. Y un primer paso fundamental en estas intervenciones implica la recopilación y evaluación de información que pueda usarse como evidencia y luego el desarrollo de estrategias legales y políticas basadas en esa evidencia [5]. La propagación y adopción de las tecnologías de comunicación (internet, teléfonos inteligentes, plataformas de redes sociales, cartografía y tecnologías geoespaciales) han transformado el proceso de documentación y acción de organizaciones enfocadas en defender los derechos humanos [11]. Un ejemplo de esta práctica son los cientos de videos de YouTube que han documentan violaciones de derechos humanos durante la guerra civil en Siria [7].

Durante la última década ha surgido un número pequeño pero importante de estudios en Interacción Humano Computadora (IHC) que se enfocan en examinar el rol de la tecnología en apoyar a las organizaciones que defienden los derechos humanos, y también en apoyar las iniciativas ciudadanas que combaten la violencia.

Algunos ejemplos son, investigar el surgimiento de "curadores" - individuos que actúan como "corresponsales de guerra" - que agregan y difunden información a grandes audiencias en las redes sociales [10]; algunos otros estudios se han centrado en entender el impacto afectivo que ha tenido el crimen en los ciudadanos [8] y comprender cómo los grupos armados de civiles conocidos como "fuerzas de autodefensa" han surgido a través de canales en línea para desafiar a los poderosos cárteles de la droga [9]. Muchos de estos grupos han adoptado roles propagandistas y estrategias de movilización en línea para definir la narrativa del conflicto armado [9].

\section{Investigación Completada}

Hasta la fecha, he estado realizando esta investigación en mi país de origen, México, que está experimentando una nueva crisis de violaciones de derechos humanos y violencia extrema. En mi investigación he utilizado métodos mixtos para examinar las prácticas de datos de las comunidades que se organizan a través de las redes sociales para abordar crisis locales y de derechos humanos, así como las prácticas de datos de las ONG que podrían beneficiarse de dichos datos al actuar sobre ellos. Los hallazgos de mi trabajo finalizado han informado el diseño de los primeros prototipos de herramientas que abordan los desafíos de movilizar el conocimiento local de las comunidades en línea para su uso en el contexto de las ONG. Mi trabajo ya se ha publicado en lugares competitivos como ACM CSCW, y los capítulos de tesis se resumen de la siguiente manera:

\subsection{Estudio 1: Entendiendo el uso de datos por activistas}

En este primer estudio me enfoque en examinar cómo las ONG que se centran en las violaciones de derechos humanos en México obtienen y traducen datos para producir información para realizar su trabajo [3].

\subsubsection{Métodos}

Realice un estudio cualitativo que consistió en entrevistar a 15 participantes que trabajan en think tanks, centros de derechos humanos y ONG de todo México.

\subsubsection{Resultados}

Como resultado de este trabajo inicial, identifiqué dos desafíos de datos clave que afectan su trabajo: datos ausentes y contradictorios. Los datos ausentes se refieren a datos abiertos sobre violaciones de derechos humanos que faltan por razones tales como la eliminación abrupta de bases de datos públicas previamente disponibles; e instancias de delitos indocumentados, incluida la violencia dirigida contra defensores de derechos humanos, la existencia de fosas comunes y ejecuciones extrajudiciales. La falta de tales datos en muchos casos significa que no existe una definición establecida para estas violaciones de derechos humanos.

Los datos contradictorios se producen cuando diferentes conjuntos de datos proporcionan diferentes estadísticas para el mismo problema. Estas discrepancias hacen que sea difícil comparar datos e interpretar la escala de ciertos delitos. Para abordar estos desafíos, la mayoría de los participantes establecieron alianzas con una gran cantidad de socios con los que luego establecieron patrones de acción a través de tres prácticas de información: informar al público, solicitar acción y desarrollar capacidad.

Estos hallazgos sugieren la urgencia de explorar mecanismos alternativos para llegar a las comunidades marginales y víctimas de violaciones de derechos humanos para recopilar sus experiencias y desarrollar herramientas que faciliten su recopilación y análisis para las $\mathrm{ONG}$.

\subsection{Estudio 2: Identificando practicas locales para abordar la seguridad pública}

En base a los resultados de mi estudio anterior, en este segundo estudio me enfoque en examinar las prácticas de datos de ciudadanos en la ciudad de México que utilizan grupos de Facebook como plataforma para denunciar delitos y compartir información relacionada con la seguridad [2]. Este estudio responde a la pregunta de investigación: ¿Cómo han adoptado las personas las plataformas de redes sociales para recopilar $e$ intercambiar información sobre delitos en contextos de alta violencia?

\subsubsection{Métodos}

Durante el transcurso de un año, me uní a varios grupos de Facebook en donde los miembros informan sobre crímenes en diferentes vecindarios de la Ciudad de México. En esos grupos realicé observaciones en línea, y también realicé un estudio cualitativo que duro cinco meses y consistió en entrevistar a dos tipos de participantes: administradores y contribuyentes. Los administradores, quienes establecen y moderan el contenido, y los contribuyentes, quienes intercambian y consumen información en esos grupos. 


\subsubsection{Resultados}

Los resultados de mi estudio indicaron que existían dos motivaciones principales que llevan a los administradores y colaboradores a participar en los grupos de Facebook que intercambian información sobre violencia: primero, recopilar y centralizar datos sobre eventos actuales en sus vecindarios para superar la desinformación y la falta de datos oficiales. Segundo, mantener un registro independiente de delitos. y su efecto en sus comunidades.

Los administradores de grupo establecen prácticas comunitarias, de datos y de acción. A través de estas prácticas, los administradores primero filtraron a los miembros de la comunidad en los sitios de Facebook. Luego, los administradores recopilaron, seleccionaron y publicaron información sobre la seguridad pública que de otro modo no se informaría. Finalmente, los administradores crearon alianzas con las partes interesadas clave para traducir esos datos en resultados procesables. A pesar del valor de los datos de fuentes colectivas de las comunidades que examiné, el hecho de que no estuvieran estructurados y fueran difíciles de verificar limitó la forma en que los administradores y contribuyentes podían usarlos para abordar problemas de delincuencia y seguridad pública. Por lo tanto, para ampliar los esfuerzos de los administradores y contribuyentes, estos deben pasar de las prácticas individuales a las sistémicas.

\subsection{Estudio 3: Explorando el uso de datos de redes sociales como evidencia para la defensa de los derechos humanos}

En mi tercer estudio, examiné informes de personas desaparecidas en México que se compartieron a través de grupos de Facebook [1]. A través de un enfoque computacional de humanos en el circuito, identifiqué siete atributos de datos que facilitan la migración de este contenido y establecen una brecha de datos entre el número de casos registrados oficialmente en la base de datos del gobierno de personas desaparecidas en comparación con las cuentas de personas desaparecidas. circulando en Facebook.

En este estudio, me concentré en los informes de personas desaparecidas porque ha habido un número creciente de personas desaparecidas desde la más reciente Guerra contra las Drogas que comenzó en México en 2006. A pesar de la atención y el escrutinio de la comunidad mundial de derechos humanos, el número exacto de personas desaparecidas sigue siendo desconocido debido a inconsistencias entre las cifras proporcionadas por los informes gubernamentales y las proporcionadas por ONG nacionales e internacionales. Este estudio responde a la pregunta de investigación: ¿Cómo se pueden identificar señales significativas en los datos de las redes sociales para su uso o aplicarlas en un contexto diferente al de las redes sociales?

\subsubsection{Métodos}

mixtos: Etnografía en línea, análisis de contenido y métodos computacionales.

\subsubsection{Resultados}

Como resultado de este estudio, desarrolle una metodología que conecta métodos cualitativos y computacionales para recopilar, analizar y catalogar datos de redes sociales para ser utilizados como evidencia para las ONG. Esta metodología operativizar la migración de datos de redes sociales a un contexto fuera de línea y consiste en tres etapas: encontrar, escalar y verificar señales de redes sociales.
Además de iniciar el desarrollo de esta metodología, como resultado de este estudio, encontramos una diferencia significativa entre los datos en la base de datos oficial de personas desaparecidas y la evidencia de redes sociales. Por lo tanto, con este estudio encontré que es posible hacer uso de datos de redes sociales para informar el trabajo de organizaciones enfocadas en reducir violaciones de derechos humanos.

\section{Propuesta de Investigación}

Hasta ahora la trayectoria de mi investigación me ha guiado a identificar que es necesario diseñar herramientas para aprovechar los datos de las redes sociales considerando sus fortalezas y limitaciones considerando los múltiples actores involucrados en la movilización de estos datos en contextos institucionales. Por lo tanto, para mi disertación propongo diseñar un kit de herramientas, que estará compuesto por una colección de recursos adaptables que facilitaran las tres etapas de la metodología que he estado desarrollando para la migración de datos de redes sociales de comunidades en línea al contexto de las ONG.

El kit de herramientas será bilingüe, de código abierto y permitirá incrementar nuestro entendimiento del potencial y las limitaciones de los datos de las redes sociales para informar sobre las narrativas, las capacidades y la experiencia locales de las comunidades que de otra manera serían difíciles de identificar.

El conjunto de herramientas que propongo responderá a la siguiente pregunta de investigación: ¿Cómo diseñamos herramientas situadas que reconozcan y apoyen los desafios presentes en la migración de datos de redes sociales a contextos institucionales?

\subsection{Detalles sobre el kit de herramientas}

El kit de herramientas estará determinado por las prácticas clave de cada una de las etapas de la metodología que he ido desarrollando:

\subsubsection{Encontrar señales}

Para esta etapa, desarrollaré un conjunto de herramientas que permitan:

1. Seleccionar una plataforma de redes sociales adecuada para recopilar datos

2. Guías cualitativas para asociar los datos de las redes sociales con el problema social que está examinando la organización y para identificar características de datos clave para registrar como evidencia.

\subsubsection{Escalado de señales}

Las herramientas de esta etapa se enfocarán en facilitar las siguientes actividades:

1. Análisis de un alto volumen de datos

2. Informar la escala a la que operan problemas particulares

3. Documentar el contexto de la producción de datos

\subsubsection{Verificación de señales}

Para la última etapa las herramientas que desarrollaré se enfocaran en las siguientes actividades:

1. Verificar la precisión de los datos clasificados

2. Facilitar la referencia cruzada con registros oficiales

\subsection{Cronología}

Realizaré la última parte de mi disertación en tres etapas (vea Tabla 1). En la primera etapa, diseñaré un prototipo del kit de herramientas. En la segunda etapa, evaluaré el kit de herramientas con organizaciones en México. En la última etapa, sintetizaré mis 
hallazgos en un conjunto de pautas y un marco basado en la evaluación con mis socios. A continuación, presentaré cada fase con más detalle.

Tabla 1. Cronología de disertación

\begin{tabular}{|l|c|}
\hline Actividad & Fechas \\
\hline $\begin{array}{l}\text { Creación de prototipo del kit } \\
\text { de herramientas }\end{array}$ & Agosto - noviembre 2021 \\
\hline Evaluación & Noviembre - diciembre 2021 \\
\hline $\begin{array}{l}\text { Sintetizar hallazgos en un } \\
\text { marco teórico }\end{array}$ & Enero - mayo 2022 \\
\hline
\end{tabular}

\section{Intención de asistir al MexIHC Graduate Consortium}

Mi intención al asistir a este consorcio es primero compartir mi investigación con colegas de Latinoamérica principalmente y explorar la posibilidad de crear colaboraciones y alianzas con investigadoras e investigadores que se encuentren interesados en usar redes sociales como fuentes alternativas de conocimiento.

En segundo lugar, para diciembre esperaría contar ya con varios prototipos así como evaluaciones iniciales del kit de herramientas que propongo. Por lo tanto, durante la conferencia me gustaría recibir retroalimentación y así mejorar el resultado de mi disertación.

Por último, quisiera asistir al consorcio para conocer a mas estudiantes en el área de interacción humano-computadora y aprender sobre los proyectos que ellas o ellos estén realizando y dependiendo del caso ofrecer también retroalimentación.

\section{Referencias}

[1] Adriana Alvarado Garcia, Matthew J. Britton, Dhairya Manish Doshi, Munmun De Choudhury, and Christopher A. Le Dantec. 2021. Data Migrations: Exploring the Use of Social Media Data as Evidence for Human Rights Advocacy. Proc. ACM Hum.-Comput. Interact.4, CSCW3, Article 268 (December 2020), 25 pages.

[2] Adriana Alvarado Garcia and Christopher A. Le Dantec. 2018. Quotidian Report: Grassroots Data Practices to Address Public Safety. Proc. ACM Hum.-Comput. Interact.2, CSCW, Article 17 (November 2018), 18 pages.
[3] Adriana Alvarado Garcia, Alyson L. Young, and Lynn Dombrowski. 2017. On Making Data Actionable: How Activists Use Imperfect Data to Foster Social Change for Human Rights Violations in Mexico. Proc. ACM Hum.Comput. Interact.1, CSCW, Article 19 (November 2017), 19 pages.

[4] danah boyd and Kate Crawford. 2012. CRITICAL QUESTIONS FOR BIG DATA. Information, Communication \& Society 15, 5 (2012), 662-679.

[5] Diane F. Orentlicher. 1990. Bearing Witness: The Art and Science of Human Rights Fact-Finding

[6] Ella McPherson. 2015. ICTs and Human Rights Practice: A report prepared for the UN Special Rapporteur on extrajudicial, summary and arbitrary executions. Centre of Governance and Human Rights, University of Cambridge (2015).

[7] Malachy Browne. 2017. YouTube Removes Videos Showing Atrocities in Syria. https://www.nytimes.com/2017/08/ 22/world/middleeast/syria-youtube-videos-isis.html

[8] Munmun De Choudhury, Andrés Monroy-Hernández, and Gloria Mark. 2014. Narco emotions: affect and desensitization in social media during the mexican drug war. In Proceedings of the SIGCHI conference on Human Factors in Computing Systems. ACM, 3563-3572. *36

[9] Saiph Savage and Andrés Monroy-Hernández. 2015. Participatory Militias: An Analysis of an Armed Movement's Online Audience. In Proceedings of the 18th ACM Conference on Computer Supported Cooperative Work \& Social Computing. ACM, 724-733.

[10] Andrés Monroy-Hernández, Emre Kiciman, Munmun De Choudhury, Scott Counts, et al. 2013. The new war correspondents: The rise of civic media curation in urban warfare. In Proceedings of the 2013 conference on Computer

[11] Sam Gregory Yvette Alberdingk Thijm Padania, Sameer and Nunez Bryan. 2011. Cameras Everywhere: Current Challenges and Opportunities at the Intersection of Human Rights, Video and Technology.

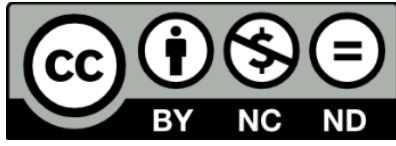

(C) 2021 by the authors. This work is licensed under the Creative Commons AttributionNonCommercial-NoDerivatives 4.0 International License. To view a copy of this license, visit http://creativecommons.org/licenses/by-nc-nd/4.0/ or send a letter to Creative Commons, PO Box 1866, Mountain View, CA 94042, USA. 